\title{
THE BOOLEAN ALGEBRA OF GALOIS ALGEBRAS
}

\author{
George Szeto and Lianyong Xue
}

Received 8 February 2002

\begin{abstract}
Let $B$ be a Galois algebra with Galois group $G, J_{g}=\{b \in B \mid b x=g(x) b$ for all $x \in$ $B$ \} for each $g \in G$, and $B J_{g}=B e_{g}$ for a central idempotent $e_{g}, B_{a}$ the Boolean algebra generated by $\left\{0, e_{g} \mid g \in G\right\}, e$ a nonzero element in $B_{a}$, and $H_{e}=\{g \in$ $\left.G \mid e e_{g}=e\right\}$. Then, a monomial $e$ is characterized, and the Galois extension $B e$, generated by $e$ with Galois group $H_{e}$, is investigated.
\end{abstract}

2000 Mathematics Subject Classification: 16S35, 16W20.

1. Introduction. The Boolean algebra of central idempotents in a commutative Galois algebra plays an important role for the commutative Galois theory (see $[1,3,6]$ ). Let $B$ be a Galois algebra with Galois group $G, C$ the center of $B$, and $J_{g}=\{b \in B \mid b x=g(x) b$ for all $x \in B\}$ for each $g \in G$. In [2], it was shown that $B J_{g}=B e_{g}$ for some idempotent $e_{g}$ of $C$. Let $B_{a}$ be the Boolean algebra generated by $\left\{0, e_{g} \mid g \in G\right\}$. Then in [5], by using $B_{a}$, the following structure theorem for $B$ was given. There exist $\left\{e_{i} \in B_{a} \mid i=\right.$ $1,2, \ldots, m$ for some integer $m\}$ and some subgroups $H_{i}$ of $G$ such that $B=$ $\oplus \sum_{i=1}^{m} B e_{i} \oplus B f$ where $f=1-\sum_{i=1}^{m} e_{i}, B e_{i}$ is a central Galois algebra with Galois group $H_{i}$ for each $i=1,2, \ldots, m$, and $B f=C f$ which is a Galois algebra with Galois group induced by and isomorphic with $G$ in case $1 \neq \sum_{i=1}^{m} e_{i}$. In [4], let $K$ be a subgroup of $G$. Then, $K$ is called a nonzero subgroup of $G$ if $\prod_{k \in K} e_{k} \neq 0$ in $B_{a}$, and $K$ is called a maximal nonzero subgroup of $G$ if $K \subset K^{\prime}$, where $K^{\prime}$ is a nonzero subgroup of $G$ such that $\prod_{k \in K} e_{k}=\prod_{k \in K^{\prime}} e_{k}$, then $K=K^{\prime}$. We note that any nonzero subgroup is contained in a unique maximal nonzero subgroup of $G$. In [4], it was shown that there exists a one-to-one correspondence between the set of nonzero monomials in $B_{a}$ and the set of maximal nonzero subgroups of $G$, and that, for a nonzero monomial $e$ in $B_{a}$ such that $H_{e} \neq\{1\}, B e$ is a central Galois algebra with Galois group $H_{e}$ if and only if $e$ is a minimal nonzero monomial in $B_{a}$. The purpose of the present paper is to characterize a monomial $e$ in $B_{a}$ in terms of the maximal nonzero subgroups of $G$. Then, the Galois extension $B e$, generated by a nonzero idempotent $e$ and by a monomial $e$ with Galois group $H_{e}$, is investigated, respectively. Let $G(e)=\{g \in G \mid g(e)=e\}$ for each $e \neq 0$ in $B_{a}$. We will show that (1) $H_{e}$ is a normal subgroup of $G(e)$, and (2) Be is a Galois extension of $(B e)^{H_{e}}$ with Galois group $H_{e}$ and $(B e)^{H_{e}}$ is a Galois extension of $(B e)^{G(e)}$ with Galois group $G(e) / H_{e}$. In particular, when $e$ is a monomial, $G(e)=N\left(H_{e}\right)$ (the normalizer 
of $H_{e}$ ), and when $e$ is an atom (a minimal nonzero element) of $B_{a}, B e$ is a central Galois algebra over $C e$ with Galois group $H_{e}$ and $C e$ is a commutative Galois algebra with Galois group $G(e) / H_{e}$. This generalizes and improves the result of the components of $B$ in [5, Theorem 3.8] for a Galois algebra.

2. Definitions and notations. Let $B$ be a ring with $1, C$ the center of $B, G$ an automorphism group of $B$ of order $n$ for some integer $n$, and $B^{G}$ the set of elements in $B$, fixed under each element in $G$. $B$ is called a Galois extension of $B^{G}$ with Galois group $G$ if there exist elements $\left\{a_{i}, b_{i}\right.$ in $\left.B, i=1,2, \ldots, m\right\}$ for some integer $m$ such that $\sum_{i=1}^{m} a_{i} g\left(b_{i}\right)=\delta_{1, g}$ for each $g \in G$. B is called a Galois algebra over $R$ if $B$ is a Galois extension of $R$ which is contained in $C$, and $B$ is called a central Galois extension if $B$ is a Galois extension of $C$. In this paper, we assume that $B$ is a Galois algebra with Galois group $G$. Let $J_{g}=\{b \in B \mid b x=g(x) b$ for all $x \in B\}$. In [2], it was shown that $B J_{g}=B e_{g}$ for some central idempotent $e_{g}$ of $B$. We denote $\left(B_{a} ; \dot{+}, \cdot\right)$, the Boolean algebra generated by $\left\{0, e_{g} \mid g \in G\right\}$, where $e \cdot e^{\prime}=e e^{\prime}$ and $e+e^{\prime}=e+e^{\prime}-e e^{\prime}$ for any $e$ and $e^{\prime}$ in $B_{a}$. An order relation $\leq$ is defined as usual, that is, $e \leq e^{\prime}$ in $B_{a}$ if $e \cdot e^{\prime}=e$. Throughout, $e+e^{\prime}$, for $e, e^{\prime} \in B_{a}$, means the sum in the Boolean algebra $\left(B_{a} ; \dot{+}, \cdot\right), H_{e}=\left\{g \in G \mid e \leq e_{g}\right\}$ for an $e \neq 0$ in $B_{a}$, and a monomial $e$ in $B_{a}$ is $\prod_{g \in S} e_{g} \neq 0$ for some $S \subset G$.

3. The Boolean algebra. In this section, we will characterize a monomial $e$ in $B_{a}$ in terms of the maximal nonzero subgroups of $G$. We begin with several lemmas.

LEMMA 3.1. Let $\left\{e_{i}, f \mid i=1,2, \ldots, m\right\}$ be given in [5, Theorem 3.8]. Then,

(1) $\left\{e_{i}, f \mid i=1,2, \ldots, m\right\}$ is the set of all minimal elements of $B_{a}$ in case $f \neq 0$,

(2) for each $e \neq 0$ in $B_{a}$, there exists a unique subset $Z_{e}$ of the set $\{1,2, \ldots, m\}$ such that $e=\sum_{i \in Z_{e}} e_{i}$ or $e=\sum_{i \in Z_{e}} e_{i}+f$.

Proof. (1) By the proof of [5, Theorem 3.8], either $e_{i}=\prod_{g \in H_{i}} e_{g}$, where $H_{i}$ is a maximum subset (subgroup) of $G$ such that $\prod_{g \in H_{i}} e_{g} \neq 0$, or $e_{i}=(1-$ $\left.\sum_{j=1}^{t} e_{j}\right) \prod_{g \in H_{i}} e_{g}$ for some $t<i$, where $H_{i}$ is a maximum subset (subgroup) of $G$ such that $\left(1-\sum_{j=1}^{t} e_{j}\right) \prod_{g \in H_{i}} e_{g} \neq 0$; so, either $e_{i}$ is a minimal element of $B_{a}$ or $e_{i}$ is a minimal element of $\left(1-\sum_{j=1}^{t} e_{j}\right) B_{a}$. Noting that any minimal element in $\left(1-\sum_{j=1}^{t} e_{j}\right) B_{a}$ is also a minimal element in $B_{a}$, we conclude that each $e_{i}$ is a minimal element in $B_{a}$. Next, we show that $f$ is also a minimal element of $B_{a}$ in case $f \neq 0$. In fact, by the proof of [5, Theorem 3.8], $e_{g} f=0$ for any $g \neq 1$ in $G$; so, for any $e \in B_{a}, e f=0$ or $e f=f$. This implies that $f$ is a minimal element of $B_{a}$ in case $f \neq 0$. Moreover, $\sum_{i=1}^{m} e_{i}+f=1$; so, $\left\{e_{i}, f \mid i=1,2, \ldots, m\right\}$ is the set of all minimal elements of $B_{a}$ in case $f \neq 0$.

(2) Since $1=\sum_{i=1}^{m} e_{i}+f$, a sum of all minimal elements of $B_{a}$, the statement is immediate. 
LEMMA 3.2. Let e be a nonzero element in $B_{a}$. Then,

(1) there exists a monomial $e^{\prime}$ of $B_{a}$ such that $e \leq e^{\prime}$ and $H_{e}=H_{e^{\prime}}$,

(2) $H_{e}$ is a maximal nonzero subgroup of $G$.

Proof. (1) For any nonzero element $e$ in $B_{a}$, let $e^{\prime}=\prod_{g \in H_{e}} e_{g}$. We claim that $e \leq e^{\prime}$ and $H_{e}=H_{e^{\prime}}$. In fact, for any $h \in H_{e}, e \leq e_{h}$; so, $e \leq \prod_{h \in H_{e}} e_{h}=e^{\prime}$. Moreover, for any $h \in H_{e}, e_{h} \geq \prod_{g \in H_{e}} e_{g}=e^{\prime}$; so, $h \in H_{e^{\prime}}$. Hence, $H_{e} \subset H_{e^{\prime}}$. On the other hand, for any $h \in H_{e^{\prime}}, e_{h} \geq e^{\prime}=\prod_{g \in H_{e}} e_{g} \geq e$; so, $h \in H_{e}$. Thus, $H_{e^{\prime}} \subset H_{e}$. Therefore, $H_{e}=H_{e^{\prime}}$.

(2) By [4, Theorem 3.2], $H_{e^{\prime}}$ is a maximal nonzero subgroup of $G$ for $e^{\prime}$ is a monomial. Hence, $H_{e}\left(=H_{e^{\prime}}\right)$ is a maximal nonzero subgroup of $G$.

Next is an expression of $H_{e}$ for a nonzero $e \in B_{a}$.

THEOREM 3.3. For any $e \neq 0$ in $B_{a}, H_{e}=\cap_{i \in Z_{e}} H_{e_{i}}$ or $H_{1}$, where $e=\sum_{i \in Z_{e}} e_{i}$ or $e=\sum_{i \in Z_{e}} e_{i}+f$ as given in Lemma 3.1(2).

Proof. We first show that for $e=e^{\prime}+e^{\prime \prime}$ for some $e^{\prime}, e^{\prime \prime} \neq 0$ in $B_{a}, H_{e}=H_{e^{\prime}} \cap$ $H_{e^{\prime \prime}}$. In fact, since $e \geq e^{\prime}$ and $e \geq e^{\prime \prime}$, we have $H_{e} \subset H_{e^{\prime}} \cap H_{e^{\prime \prime}}$. Conversely, for any $g \in H_{e^{\prime}} \cap H_{e^{\prime \prime}}, e_{g} \geq e^{\prime}$ and $e_{g} \geq e^{\prime \prime} ;$ so, $e_{g} \geq e^{\prime}+e^{\prime \prime}=e$. Hence, $g \in H_{e}$; so, $H_{e}=$ $H_{e^{\prime}} \cap H_{e^{\prime \prime}}$. Therefore, by induction, if $e=\sum_{i \in Z_{e}} e_{i}$, then $H_{e}=\cap_{i \in Z_{e}} H_{e_{i}}$. Now, by Lemma 3.1, for any $e \neq 0$ in $B_{a}, e=\sum_{i \in Z_{e}} e_{i}$ or $e=\sum_{i \in Z_{e}} e_{i}+f$. Similarly, if $e=\sum_{i \in Z_{e}} e_{i}+f$, then $H_{e}=H_{\left(\sum_{i \in Z_{e}} e_{i}\right)+f}=\left(\cap_{i \in Z_{e}} H_{e_{i}}\right) \cap H_{f}$. But, for $g \in G$ such that $e_{g} \neq 1, e_{g} f=0$; so, $H_{f}=H_{1}$. Therefore, $H_{e}=\left(\cap_{i \in Z_{e}} H_{e_{i}}\right) \cap H_{1}=H_{1}$ for $H_{1} \subset H_{e_{i}}$ for each $i$.

We observe that there exist some $e \neq 0$ such that $H_{e}=\cap_{i \in Z_{e}} H_{e_{i}}$ and $H_{e} \subset H_{e_{j}}$ for some $j \notin Z_{e}$, and that not all $e \neq 0$ are monomials. Next, we identify which element $e \neq 0$ in $B_{a}$ is a monomial. Two characterizations are given. We begin with a definition.

DEFINITION 3.4. An $e \neq 0$ in $B_{a}$ is called a maximal $G$-element if $H_{e} \neq H_{1}$ and, for any $e^{\prime} \in B_{a}$ such that $e \leq e^{\prime}$ and $H_{e}=H_{e^{\prime}}, e=e^{\prime}$.

LEMMA 3.5. (1) If $e \neq 0$ such that ef $=0$, then $e=\sum_{i \in Z_{e}} e_{i}$.

(2) If $e$ is a monomial, $e=\prod_{g \in S} e_{g}$ for some $S \subset G$, then $e=1$ or $e=\sum_{i \in Z_{e}} e_{i}$.

Proof. (1) By Lemma 3.1, $e=\sum_{i \in Z_{e}} e_{i}$ or $e=\sum_{i \in Z_{e}} e_{i}+f$. If $e \neq \sum_{i \in Z_{e}} e_{i}$, then $e=\sum_{i \in Z_{e}} e_{i}+f$ and $f \neq 0$. But then, $f=\left(\sum_{i \in Z_{e}} e_{i}+f\right) f=e f=0$. This is a contradiction. Hence, $e=\sum_{i \in Z_{e}} e_{i}$.

(2) In case $e=1$, we are done. In case $e \neq 1$. Since $e_{g} f=0$ for each $g \in G$ such that $e_{g} \neq 1, e f=\prod_{g \in S} e_{g} f=0$. Thus, by (1), $e=\sum_{i \in Z_{e}} e_{i}$.

THEOREM 3.6. Keeping the notations of Lemma 3.1 for any $e \neq 0,1$ in $B_{a}$, the following statements are equivalent:

(1) $e=\prod_{g \in S} e_{g}$ for some $S \subset G$, a monomial in $B_{a}$;

(2) $e$ is a maximal $G$-element in $B_{a}$; 
(3) $e=\sum_{i \in Z_{e}} e_{i}$ where $\left\{e_{i} \mid i \in Z_{e}\right\}$ are all atoms such that $H_{e} \subset H_{e_{i}}$ and $H_{e} \neq H_{1}$.

Proof. (1) $\Rightarrow(2)$. Since $e$ is a monomial and $e \neq 1, e=\prod_{g \in H_{e}} e_{g}$ where $e_{g} \neq 1$ for some $g \in H_{e}$. Thus, $H_{e} \neq H_{1}$. Next, for any $e^{\prime}$ such that $e \leq e^{\prime}$ and $H_{e}=H_{e^{\prime}}$,

$$
e \leq e^{\prime} \leq \prod_{g \in H_{e^{\prime}}} e_{g}=\prod_{g \in H_{e}} e_{g}=e .
$$

Hence, $e=e^{\prime}$. This implies that $e$ is a maximal $G$-element in $B_{a}$.

$(2) \Rightarrow(1)$. Let $e$ be a maximal $G$-element and $e^{\prime}=\prod_{g \in H_{e}} e_{g}$. Then, by Lemma 3.2, $e \leq e^{\prime}$ and $H_{e}=H_{e^{\prime}}$. But $e$ is a maximal $G$-element; so, $e=e^{\prime}$ which is a monomial.

(1) $\Rightarrow$ (3). By Lemma 3.5, $e=\sum_{i \in Z_{e}} e_{i}$. Now, let $e_{j}$ be an atom such that $H_{e} \subset$ $H_{e_{j}}$. Then, $e_{j} \leq \prod_{g \in H_{e_{j}}} e_{g} \leq \prod_{g \in H_{e}} e_{g}$. But, by hypothesis, $e$ is a monomial; so, $e=\prod_{g \in H_{e}} e_{g}$. Hence, $e_{j} \leq e$. This implies that $e_{j}$ is a term in $e$. Thus, $e=$ $\sum_{i \in Z_{e}} e_{i}$ where $\left\{e_{i} \mid i \in Z_{e}\right\}$ are all atoms such that $H_{e} \subset H_{e_{i}}$. Moreover, since $e=\prod_{g \in S} e_{g} \neq 1$, there exists $g \in G$ such that $e \leq e_{g} \neq 1$. Thus, $g \in H_{e}$ and $g \notin H_{1}$. Therefore, $H_{e} \neq H_{1}$.

(3) $\Rightarrow$ (1). Let $e^{\prime}=\prod_{g \in H_{e}} e_{g}$. Then, by Lemma 3.2, $e \leq e^{\prime}$ and $H_{e}=H_{e^{\prime}}$. Since $H_{e} \neq H_{1}, H_{e^{\prime}} \neq H_{1}$. Also, since $e^{\prime}$ is a monomial, $e^{\prime}=\sum_{j \in Z_{e^{\prime}}} e_{j}$ by Lemma 3.5(2). Now, suppose that $e \neq e^{\prime}$. Then, there is a $j \in Z_{e^{\prime}}$ but $j \notin Z_{e}$, that is, $e_{j}$ is a term of $e^{\prime}=\sum_{j \in Z_{e^{\prime}}} e_{j}$ but not a term of $e=\sum_{i \in Z_{e}} e_{i}$. But then, $H_{e}=H_{e^{\prime}}=\cap_{j \in Z_{e^{\prime}}} H_{e_{j}} \subset$ $H_{e_{j}}$ such that $j \notin Z_{e}$. This contradicts the hypothesis that $e=\sum_{i \in Z_{e}} e_{i}$ where $\left\{e_{i} \mid i \in Z_{e}\right\}$ are all atoms such that $H_{e} \subset H_{e_{i}}$. Thus, $e=e^{\prime}$ which is a monomial in $B_{a}$.

4. Galois extensions. In [5], it was shown that Be is a central Galois algebra with Galois group $H_{e}$ for any atom $e \neq f$ of $B_{a}$. Also, for any $e \neq 0$ in $B_{a}$, $B e$ is a Galois extension of $(B e)^{G(e)}$ with Galois group $\left.G(e)\right|_{B e} \cong G(e)$ where $G(e)=\{g \in G \mid g(e)=e\}$ (see [5, Lemma 3.7]). In this section, we are going to show that, for any $e \neq 0$ in $B_{a}$ (not necessary an atom), (1) $H_{e}$ is a normal subgroup of $G(e)$, and (2) $B e$ is a Galois extension of $(B e)^{H_{e}}$ with Galois group $H_{e}$ and $(B e)^{H_{e}}$ is a Galois extension of $(B e)^{G(e)}$ with Galois group $G(e) / H_{e}$. This generalizes and improves the result for $B e$ when $e$ is an atom of $B_{a}$ as given in [5, Theorem 3.8]. In particular, for a monomial $e, G(e)=N\left(H_{e}\right)$, the normalizer of $H_{e}$ in $G$.

LEMMA 4.1. Let $e \neq 0$ in $B_{a}$. Then, $H_{e}$ is a normal subgroup of $G(e)$ where $G(e)=\{g \in G \mid g(e)=e\}$.

PROOF. We first claim that $H_{e} \subset G(e)$. In fact, by Lemma 3.1, for any $e \neq 0$ in $B_{a}$, there exists a unique subset $Z_{e}$ of the set $\{1,2, \ldots, m\}$ such that $e=\sum_{i \in Z_{e}} e_{i}$ or $e=\sum_{i \in Z_{e}} e_{i}+f$ where $e_{i}$ are given in Lemma 3.1. Moreover, for each $i$, 
$e_{i}=\prod_{h \in H_{e_{i}}} e_{h}$ or $e_{i}=\left(1-\sum_{j=1}^{t} e_{j}\right) \prod_{g \in H_{e_{i}}} e_{g}$ for some $t<i$. Noting that $g$ permutes the set $\left\{e_{i} \mid i=1,2, \ldots, t\right\}$ for each $g \in G$ by the proof of [5, Theorem 3.8], we have, for each $g \in G$,

$$
g\left(e_{i}\right)=g\left(\prod_{h \in H_{e_{i}}} e_{h}\right)=\prod_{h \in H_{e_{i}}} e_{g h g^{-1}} \geq \prod_{h \in H_{e_{i}}} e_{g} e_{h} e_{g^{-1}}=e_{g} e_{i} e_{g^{-1}}
$$

or

$$
\begin{aligned}
g\left(e_{i}\right) & =g\left(\left(1-\sum_{j=1}^{t} e_{j}\right) \prod_{h \in H_{e_{i}}} e_{h}\right)=\left(1-\sum_{j=1}^{t} e_{j}\right) \prod_{h \in H_{e_{i}}} e_{g h g^{-1}} \\
& \geq\left(1-\sum_{j=1}^{t} e_{j}\right) \prod_{h \in H_{e_{i}}} e_{g} e_{h} e_{g^{-1}} \\
& =e_{g}\left(\left(1-\sum_{j=1}^{t} e_{j}\right) \prod_{h \in H_{e_{i}}} e_{h}\right) e_{g^{-1}}=e_{g} e_{i} e_{g^{-1}}
\end{aligned}
$$

Now, in case $e=\sum_{i \in Z_{e}} e_{i}$, for any $h \in H_{e}$,

$$
e=e_{h} e e_{h^{-1}}=\sum_{i \in Z_{e}} e_{h} e_{i} e_{h^{-1}} \leq \sum_{i \in Z_{e}} h\left(e_{i}\right)=h(e) .
$$

Thus, $h(e)=e$ using Lemma 3.1(2). Noting that $g$ permutes the set $\left\{e_{i} \mid i=\right.$ $1,2, \ldots, m\}$ for each $g \in G$, we have $g(f)=f$ for each $g \in G$. Thus, we have $h(e)=e$ for each $h \in H_{e}$ in case $e=\sum_{i \in Z_{e}} e_{i}+f$. This proves that $H_{e} \subset G(e)$. Next, we show that $H_{e}$ is a normal subgroup of $G(e)$. Since for each $g \in G$, $g\left(e_{i}\right)$ is also an atom, $g(e)=e$ (i.e., $\left.g \in G(e)\right)$ implies that $g$ permutes the set $\left\{e_{i} \mid i \in Z_{e}\right\}$. Therefore, for each $i \in Z_{e}, g\left(e_{i}\right)=e_{j}$ and $g H_{e_{i}} g^{-1}=H_{e_{j}}$ for some $j \in Z_{e}$. But, by Theorem 3.3, $H_{e}=\cap_{i \in Z_{e}} H_{e_{i}}$ (or $H_{e}=H_{1}$ which is normal); so, for any $g \in G(e), g H_{e} g^{-1}=g\left(\cap_{i \in Z_{e}} H_{e_{i}}\right) g^{-1}=\cap_{i \in Z_{e}} g H_{e_{i}} g^{-1}=\cap_{j \in Z_{e}} H_{e_{j}}=H_{e}$. Therefore, $H_{e}$ is a normal subgroup of $G(e)$.

THEOREM 4.2. Let e be a nonzero element in $B_{a}$. Then,

(1) Be is a Galois extension of $(B e)^{G(e)}$ with Galois group $G(e)$,

(2) Be is a Galois extension of (Be $)^{H_{e}}$ with Galois group $H_{e}$ and $(B e)^{H_{e}}$ is a Galois extension of $(B e)^{G(e)}$ with Galois group $G(e) / H_{e}$.

Proof. (1) Since $B$ is a Galois algebra with Galois group $G, B$ is a Galois extension with Galois group $G(e)$. But $g(e)=e$ for each $g \in G(e)$; so, by [5, Lemma 3.7], Be is a Galois extension of $(B e)^{G(e)}$ with Galois group $G(e)$.

(2) Clearly, $B e$ is a Galois extension of $(B e)^{H_{e}}$ with Galois group $H_{e}$ by part (1). Next, we claim that $\left|H_{e}\right|$, the order of $H_{e}$, is a unit in Be. In fact, by [5, Theorem 3.8], for each atom $e_{i}$ of $B_{a}, B e_{i}$ is a central Galois algebra over $C e_{i}$ with Galois group $H_{e_{i}}$; so, $\left|H_{e_{i}}\right|$, the order of $H_{e_{i}}$, is a unit in $B e_{i}$ (see [2, Corollary 3]). Hence, $\left|H_{e}\right|\left(=\left|\cap H_{e_{i}}\right|\right)$ is a unit in Be if $e=\sum_{i \in Z_{e}} e_{i}$. If $e=\sum_{i \in Z_{e}} e_{i}+f$ and $f \neq 0$, then $H_{e}=H_{1}=\left\{g \in G \mid e_{g}=1\right\}=\{g \in G \mid g(c)=c$ for each $c \in C\}$. Hence, by 
[2, Proposition 5], $\left|H_{e}\right|$ is a unit in $B$. Thus, $(B e)^{H_{e}}$ is a Galois extension of $(B e)^{G(e)}$ with Galois group $G(e) / H_{e}$ for $H_{e}$ is a normal subgroup of $G(e)$ by Lemma 4.1.

Lemma 4.1 shows that, for any nonzero element $e$ in $B_{a}, G(e)$ is contained in (not necessarily equal to) the normalizer $N\left(H_{e}\right)$ of $H_{e}$ in $G$. Next, we want to show that $G(e)=N\left(H_{e}\right)$ when $e$ is a monomial. Consequently, for any nonzero element $e$ in $B_{a}, B e$ is embedded in a Galois extension $B e^{\prime}$ of $\left(B e^{\prime}\right)^{H_{e}}$ with the same Galois group $H_{e}$, and $\left(B e^{\prime}\right)^{H_{e}}$ is a Galois extension of $\left(B e^{\prime}\right)^{G\left(e^{\prime}\right)}$ with Galois group $G\left(e^{\prime}\right) / H_{e}$ such that $G\left(e^{\prime}\right)=N\left(H_{e}\right)$ for some monomial $e^{\prime}$ in $B_{a}$.

LEMMA 4.3. Let e be a nonzero element in $B_{a}$. Then, there exists a monomial $e^{\prime}$ in $B_{a}$ such that $e \leq e^{\prime}, H_{e}=H_{e^{\prime}}$, and $N\left(H_{e}\right)=G\left(e^{\prime}\right)$ where $G\left(e^{\prime}\right)=\{g \in G \mid$ $\left.g\left(e^{\prime}\right)=e^{\prime}\right\}$ and $N\left(H_{e}\right)$ is the normalizer of $H_{e}$ in $G$.

Proof. By Lemma 3.2, there exists a monomial $e^{\prime}$ in $B_{a}$ such that $e \leq e^{\prime}$ and $H_{e}=H_{e^{\prime}}$; so, it suffices to show that $N\left(H_{e}\right)=G\left(e^{\prime}\right)$. For any $g \in N\left(H_{e}\right), g \in$ $N\left(H_{e^{\prime}}\right)$; so, by Theorem 3.3, $H_{e^{\prime}}=g H_{e^{\prime}} g^{-1}=g\left(\cap_{i \in Z_{e^{\prime}}} H_{e_{i}}\right) g^{-1}=\cap_{i \in Z_{e^{\prime}}} g H_{e_{i}} g^{-1}=$ $\cap_{i \in Z_{e^{\prime}}} H_{g\left(e_{i}\right)}=H_{\sum_{i \in Z_{e^{\prime}}} g\left(e_{i}\right)}=H_{g\left(e^{\prime}\right)}$. Noting that $e^{\prime}$ is a monomial, we have $g\left(e^{\prime}\right)=$ $e^{\prime}$ by Lemma 3.2, that is, $g \in G\left(e^{\prime}\right)$. This implies that $N\left(H_{e}\right) \subset G\left(e^{\prime}\right)$. Conversely, $G\left(e^{\prime}\right) \subset N\left(H_{e^{\prime}}\right)$ by Lemma 4.1. But $H_{e}=H_{e^{\prime}}$; so, $G\left(e^{\prime}\right) \subset N\left(H_{e^{\prime}}\right)=$ $N\left(H_{e}\right)$. Therefore, $N\left(H_{e}\right)=G\left(e^{\prime}\right)$.

THEOREM 4.4. Let e be a nonzero element in $B_{a}$. Then, there exists a monomial $e^{\prime}$ in $B_{a}$ such that Be is embedded in $B e^{\prime}, B e^{\prime}$ is a Galois extension of $\left(B e^{\prime}\right)^{H_{e}}$ with Galois group $H_{e}$, and $\left(B e^{\prime}\right)^{H_{e}}$ is a Galois extension of $\left(B e^{\prime}\right)^{N\left(H_{e}\right)}$ with Galois group $N\left(H_{e}\right) / H_{e}$.

Proof. By Lemma 4.3, there exists a monomial $e^{\prime}$ in $B_{a}$ such that $e \leq e^{\prime}$, $H_{e}$ is a normal subgroup of $G\left(e^{\prime}\right)$, and $N\left(H_{e}\right)=G\left(e^{\prime}\right)$. Hence, $B e \subset B e^{\prime}$. But $B e^{\prime}$ is a Galois extension of $\left(B e^{\prime}\right)^{H_{e^{\prime}}}$ with Galois group $H_{e^{\prime}}$ and $\left(B e^{\prime}\right)^{H_{e^{\prime}}}$ is a Galois extension of $\left(B e^{\prime}\right)^{G\left(e^{\prime}\right)}$ with Galois group $G\left(e^{\prime}\right) / H_{e^{\prime}}$ by Theorem 4.2; so, Theorem 4.4 holds.

ACKNOWLEDGments. This paper was written under the support of a Caterpillar Fellowship at Bradley University, and the authors would like to thank the Caterpillar Inc. for that support.

\section{REFERENCES}

[1] F. DeMeyer, Separable polynomials over a commutative ring, Rocky Mountain J. Math. 2 (1972), no. 2, 299-310.

[2] T. Kanzaki, On Galois algebra over a commutative ring, Osaka J. Math. 2 (1965), 309-317.

[3] G. Szeto, A characterization of Azumaya algebras, J. Pure Appl. Algebra 9 (1976/1977), no. 1, 65-71.

[4] G. Szeto and L. Xue, The Boolean algebra and central Galois algebras, Int. J. Math. Math. Sci. 28 (2001), no. 4, 237-242. 
[5] _ The structure of Galois algebras, J. Algebra 237 (2001), no. 1, 238-246.

[6] O. E. Villamayor and D. Zelinsky, Galois theory with infinitely many idempotents, Nagoya Math. J. 35 (1969), 83-98.

George Szeto: Department of Mathematics, Bradley University, Peoria, IL 61625, USA E-mail address: szeto@hi11top.brad1ey.edu

Lianyong Xue: Department of Mathematics, Bradley University, Peoria, IL 61625, USA E-mail address: 1xue@hi11 top.brad1ey.edu 


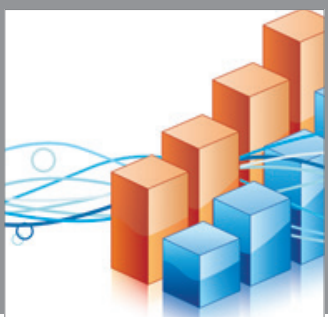

Advances in

Operations Research

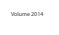

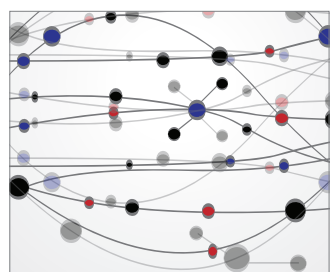

\section{The Scientific} World Journal
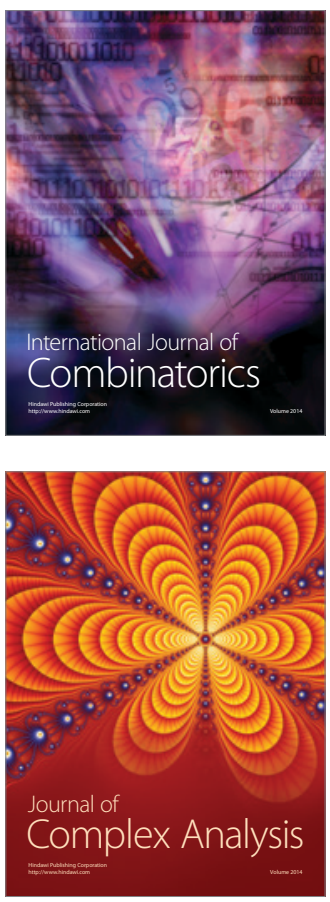

International Journal of

Mathematics and

Mathematical

Sciences
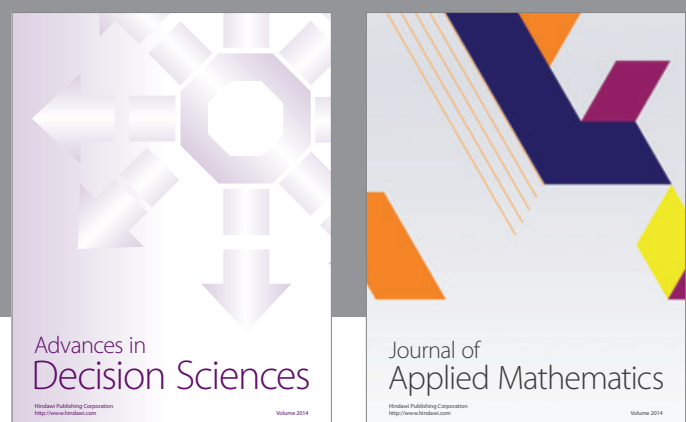

Journal of

Applied Mathematics
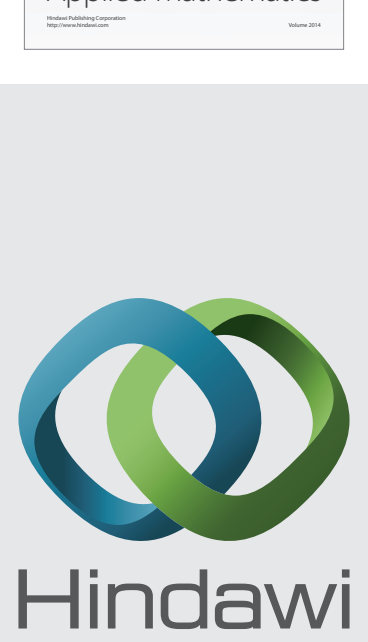

Submit your manuscripts at http://www.hindawi.com
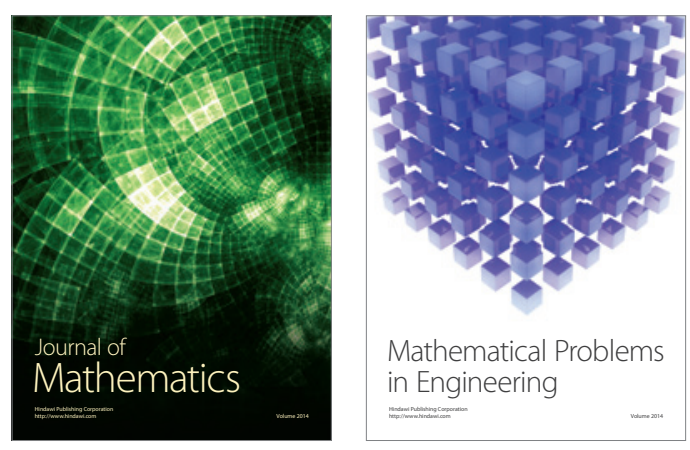

Mathematical Problems in Engineering
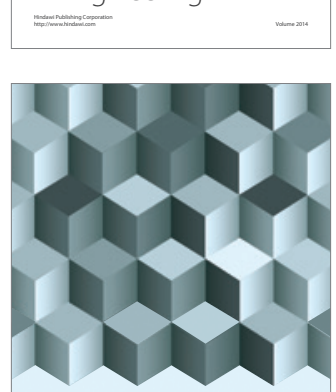

Journal of

Function Spaces
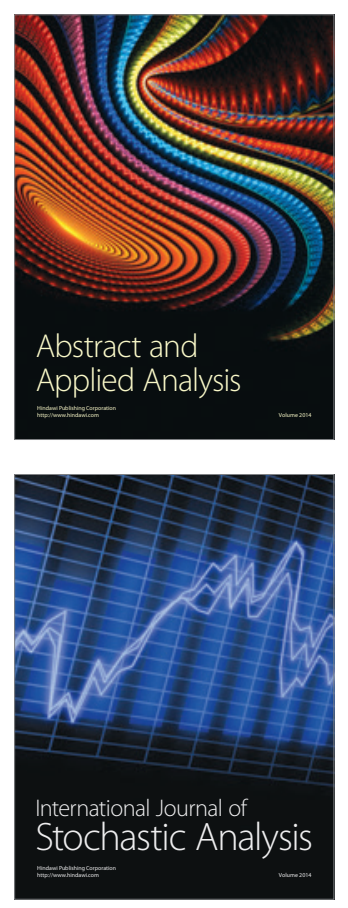

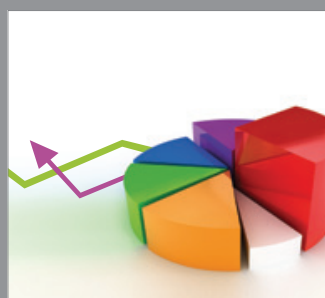

ournal of

Probability and Statistics

Promensencen
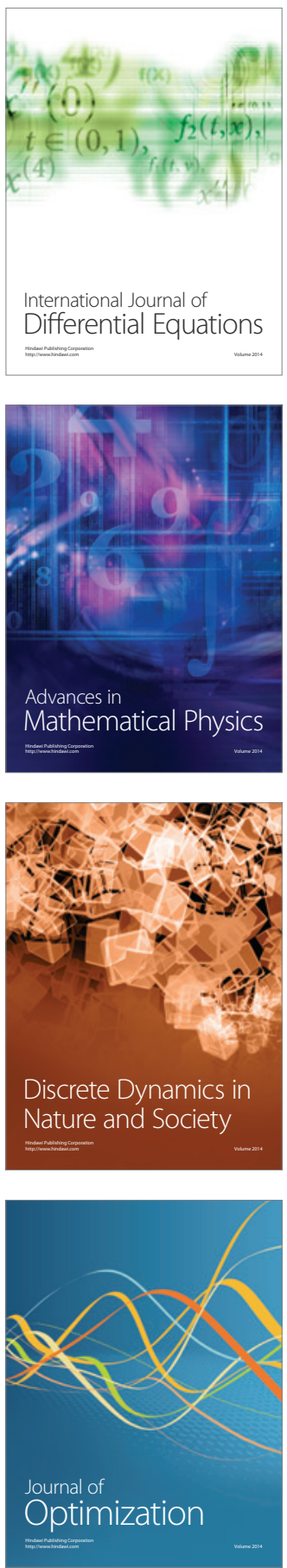